\title{
Studies on the Development and Behavior of the Dystrophic Growth Cone, the Hallmark of Regeneration Failure, in an In Vitro Model of the Glial Scar and after Spinal Cord Injury
}

\author{
Veronica J. Tom, ${ }^{1}$ Michael P. Steinmetz, ${ }^{2}$ Jared H. Miller, ${ }^{1}$ Catherine M. Doller, ${ }^{1}$ and Jerry Silver ${ }^{1}$ \\ ${ }^{1}$ Department of Neurosciences, Case Western Reserve University, Cleveland, Ohio 44106, and ${ }^{2}$ Department of Neurosurgery, Cleveland Clinic Foundation, \\ Cleveland, Ohio 44106
}

\begin{abstract}
We have developed a novel in vitro model of the glial scar that mimics the gradient of proteoglycan found in vivo after spinal cord injury. In this model, regenerated axons from adult sensory neurons that extended deeply into the gradient developed bulbous, vacuolated endings that looked remarkably similar to dystrophic endings formed in vivo. We demonstrate that despite their highly abnormal appearance and stalled forward progress, dystrophic endings are extremely dynamic both in vitro and in vivo after spinal cord injury. Time-lapse movies demonstrated that dystrophic endings continually send out membrane veils and endocytose large membrane vesicles at the leading edge, which were then retrogradely transported to the rear of the "growth cone." This direction of movement is contrary to membrane dynamics that occur during normal neurite outgrowth. As further evidence of this motility, dystrophic endings endocytosed large amounts of dextran both in vitro and in vivo. We now have an in vitro model of the glial scar that may serve as a potent tool for developing and screening potential treatments to help promote regeneration past the lesion in vivo.
\end{abstract}

Key words: dorsal root ganglion; dystrophic; growth cone; injury; proteoglycan; regeneration

\section{Introduction}

After spinal cord injury, a glial scar forms that poses a major impediment to CNS regeneration (Silver and Miller, 2004). In the region of forming scar tissue, the ends of the regenerating axons cease extending and become swollen and distorted into various bizarrely shaped "growth cones" that can remain for years within axon tracts (Li and Raisman, 1995; Houle and Jin, 2001; Kwon et al., 2002). Ramón y Cajal (1928) believed that these "sterile clubs" or "dystrophic endballs" were a relatively quiescent, final resting state of the frustrated growth cone. Thus, it has long been theorized that dystrophic axons are incapable of robust regeneration. Few studies, however, have sought to elucidate why these endings form in the scar and what motile behaviors, if any, they are capable of producing.

It is now known that in the region of forming scar tissue, several classes of growth inhibitory molecules are upregulated, including the family of extracellular matrix molecules known as chondroitin/keratan sulfate proteoglycans (PGs) that appears rapidly after injury in the vicinity of blood-brain barrier breakdown (Fitch and Silver, 1997; Morgenstern et al., 2002; Jones et al., 2003; Tang et al., 2003). PGs are organized in a crude gradient

Received March 17, 2004; revised June 4, 2004; accepted June 4, 2004.

This work was supported by National Institute of Neurological Disorders and Stroke Grant NS 25713, the Daniel Heumann Fund, and the Brumagin Memorial Fund. We are grateful to Rui Wang and Dr. Robert Miller for generously providing oligodendrocytes and Dr. Marie Filbin for generously providing purified myelin membrane. We also thank Dr. John Houle, Kevin Horn, and Alicia Yonkof for helpful comments on this manuscript.

Correspondence should be addressed to Dr. Jerry Silver, Case Western Reserve University, Department of Neurosciences, 10900 Euclid Avenue, Cleveland, 0H 44106. E-mail: jxs10@cwru.edu.

DOI:10.1523/JNEUROSCI.0994-04.2004

Copyright $\odot 2004$ Society for Neuroscience $\quad$ 0270-6474/04/246531-09\$15.00/0 with the lowest concentrations in the lesion penumbra and the highest in the epicenter (Davies et al., 1999; Fitch et al., 1999). Interestingly, both growth-inhibiting and growth-promoting ECM molecules increase simultaneously in reactive astroglia. The inhibitory ECM components are more dramatically upregulated, however, blocking the intrinsic potential of the reactive glial cells to support axonal regeneration via laminin (McKeon et al., 1991). Microtransplantation experiments showed that adult sensory neurons have a robust capacity for regeneration in degenerating white matter when placed away from the lesion. Once the regenerating fibers reach the vicinity of the injury site, they are capable of struggling into the lesion penumbra but eventually cease extending and become dystrophic as they penetrate deeply into areas of highest PG concentration (Davies et al., 1999; Grimpe and Silver, 2004). Treatments that reduce or remove the inhibitory sugar chains of PGs have recently been shown to enhance regeneration and provide some functional recovery after injury (Moon et al., 2001; Bradbury et al., 2002; Mayes and Houle, 2003; Tester et al., 2003; Grimpe and Silver, 2004), illustrating their importance in regeneration failure; however, previous in vitro models that have used sharp-edged (i.e., stripe) substrate assays to examine the effects of PGs on axons induce either growth cone turning or collapse, but not dystrophy (Snow et al., 1990).

We wanted to directly determine whether a crude gradient of PGs is sufficient to produce dystrophic endings in regenerating adult axons. To this end, we developed a novel in vitro system that forces regenerating axons of adult sensory neurons to cope with a spot gradient of the PG aggrecan mixed with laminin. As fibers 
extend into the gradient, bulbous multivesiculated endings remarkably similar to those described by Ramón y Cajal (1928) are formed in this glial scar model. We show for the first time that PGs can lead to growth cone dystrophy and that, surprisingly, supposedly sterile dystrophic endings are extraordinarily dynamic.

\section{Materials and Methods}

Preparation of aggrecan-laminin spot gradient coverslips. Glass coverslips coated with poly-lysine and nitrocellulose were spotted with $2 \mu \mathrm{l}$ of a solution of aggrecan $(0.7 \mathrm{mg} / \mathrm{ml}$; Sigma, St. Louis, MO) and laminin (5 $\mu \mathrm{g} / \mathrm{ml}$; Biomedical Technologies, Stoughton, MA) in calcium- and magnesium-free HBSS (CMF) (Invitrogen, Gaithersburg, MD). These concentrations were decided on after we observed the effects of varying concentrations of both aggrecan $(0.5-1.4 \mathrm{mg} / \mathrm{ml})$ and laminin (5-25 $\mu \mathrm{g} / \mathrm{ml}$ ) on dorsal root ganglion (DRG) neurites: $0.7 \mathrm{mg} / \mathrm{ml}$ of aggrecan and $5 \mu \mathrm{g} / \mathrm{ml}$ of laminin resulted in consistently good cell attachment with limited neurite outgrowth but no crossing of the inhibitory rim. The other concentrations tested resulted in either too few cells adhering to the substrate (with the higher concentrations of aggrecan) or too much outgrowth and crossing of the rim (with higher concentrations of laminin). Control spots of laminin mixed with either bovine serum albumin (BSA) $(0.7 \mathrm{mg} / \mathrm{ml}$; Sigma $)$ or fibronectin ( $10 \mu \mathrm{g} / \mathrm{ml}$; Biomedical Technologies) were also made. After the spots were allowed to air dry, the coverslips were completely covered with laminin $(5 \mu \mathrm{g} / \mathrm{ml})$ in CMF and kept at $37^{\circ} \mathrm{C}$ until just before cell plating $(\sim 5 \mathrm{hr})$.

$D R G$ dissociation. DRGs were harvested as described previously (Davies et al., 1999). Briefly, DRGs were dissected out of adult Sprague Dawley female rats (Zivic Miller). After the roots were trimmed, the ganglia were incubated in dispase-collagenase in CMF. The ganglia were rinsed several times in fresh CMF and gently triturated. After several low-speed spins $(\sim 370 \times g)$, the cells were plated at a density of 6000 cells per coverslip (for high-density cultures; see Fig. 1c) and 2000 cells per coverslip (4000 cells/ml) in Neurobasal A/B27 (Invitrogen).

Time-lapse video microscopy. Neuronal cultures used for time-lapse microscopy were prepared as above with the exception that the cultures were grown on culture dishes specifically designed for use with the Delta-T culture system (Bioptechs) on an inverted microscope (Zeiss Axiovert $405 \mathrm{M}$ ). Just before imaging, the media was changed to Neurobasal A/B27 with $10 \mathrm{~mm}$ 4-morpholinepropanesulfonic acid, $\mathrm{pH}$ 7.4. Phase-contrast images were taken using a $100 \times$ oil-immersion objective every minute using the imaging program MetaMorph. The stage $x-y$ coordinates of the imaged growth cones were noted for future reference. To measure the rate of outgrowth of growth cones, the difference between the initial and final locations of growth cones $(n=8$ for each group) was calculated and divided by the time elapsed between the two time points. Statistical relevance was determined using a Student's $t$ test. Local motility at the growth cone was quantified using time-lapse movies of three different growth cones. The individual image files comprising the movies were isolated, and the distance from a fixed point in the substrate to the farthest end of the growth cone was measured every 5 min over a $2 \mathrm{hr}$ period. The appearance or disappearance of a membrane veil was also noted at each time point.

Immunohistochemistry and microscopy. Cultures were immersion fixed in 4\% paraformaldehyde in PBS for $30 \mathrm{~min}$. After several rinses in fresh PBS, the coverslips were incubated in blocking solution ( $5 \%$ normal goat serum, $0.1 \%$ BSA, $0.1 \%$ Triton X-100 in PBS for intracellular proteins) for $1 \mathrm{hr}$ at room temperature and then incubated overnight at $4^{\circ} \mathrm{C}$ in primary antibody. The primary antibodies used were anti- $\beta$-tubulin type III (1:1000; Sigma), CS56 (1:200; Sigma), and anti-laminin-1 (1:2000; Biomedical Technologies). The coverslips were rinsed several times in PBS and then incubated in the appropriate secondary antibody (Jackson ImmunoResearch, West Grove, PA, or Molecular Probes, Eugene, OR) for $1 \mathrm{hr}$ at room temperature. They were rinsed again, mounted on glass slides in Citifluor (Ted Pella), and sealed with nail polish. To obtain spinal cord sections, rats were transcardially perfused with ice-cold $4 \%$ paraformaldehyde in PBS, and the spinal cords were dissected out. After the tissue was postfixed in $4 \%$ paraformaldehyde overnight at $4^{\circ} \mathrm{C}, 50 \mu \mathrm{m}$ sections were cut on a vibratome and processed for immunohistochemistry as described above. Specimens were examined using a Leitz Orthoplan 2 fluorescence microscope and a Zeiss LSM 410 confocal microscope.

Scanning electron microscopy of growth cones. DRG cultures were grown on glass coverslips as described above. Some cultures were grown on either aggrecan-laminin-spotted substrates, and others were grown on laminin-only substrates. Five days later, the cultures were fixed in 3\% glutaraldehyde and processed for scanning electron microscopy. Briefly, the coverslips were rinsed several times, postfixed for $1 \mathrm{hr}$ in $1 \% \mathrm{OsO}_{4}$, rinsed, and then exposed to $1 \%$ tannic acid for $30 \mathrm{~min}$. After several rinses, the samples were dehydrated in ethanol and exposed to hexamethyldisilazane. They were then sputter coated and viewed with a scanning electron microscope.

Quantification of the laminin and aggrecan gradients. To quantify the laminin and aggrecan gradients, coverslips were spotted with $0.7 \mathrm{mg} / \mathrm{ml}$ of aggrecan and $5 \mu \mathrm{g} / \mathrm{ml}, 10 \mu \mathrm{g} / \mathrm{ml}$, or $25 \mu \mathrm{g} / \mathrm{ml}$ of laminin. The coverslips were fixed with $4 \%$ paraformaldehyde and stained with the CS56 antibody (recognizing 4- and 6-sulfated proteoglycans) and a rabbit antilaminin-1 antibody (Biomedical Technologies) as described above. Images of the spots containing the rim were obtained using a digital camera while using a constant exposure time. Average pixel intensity was quantified at $10 \mu \mathrm{m}$ intervals between the outer edge of the spot and $70 \mu \mathrm{m}$ in toward the center of the spot, thereby spanning the width of the rim (total of eight measurements per spot). Eight spots were quantified per group, for a total of 24 spots.

To quantify the number of neurites crossing the rim because of increasing laminin concentrations, DRGs were grown on coverslips spotted with $0.7 \mathrm{mg} / \mathrm{ml}$ of aggrecan and $5 \mu \mathrm{g} / \mathrm{ml}, 10 \mu \mathrm{g} / \mathrm{ml}$, or $25 \mu \mathrm{g} / \mathrm{ml}$ of laminin. Five days later, the coverslips were fixed and stained for $\beta$-tubulin III. The number of neurites that completely crossed the rim region was counted per spot ( 8 spots per group, for a total of 24 spots). The average number of neurites crossing was calculated per group. The data were analyzed for statistical relevance using ANOVA followed by a post hoc Tukey test.

Dextran labeling of dystrophic endings in vitro. Glass coverslips with the aggrecan spot gradient were prepared as mentioned above. Dissociated DRGs ( $\sim 2000$ cells) were plated onto the coverslips and maintained for $5 \mathrm{~d}$. Glass coverslips were also prepared in a similar manner, with the exception that no aggrecan spot was applied (i.e., laminin only). A lowdensity DRG suspension ( $\sim 250$ cells per coverslip) was plated onto these coverslips for laminin-only growth cone controls. These cultures were also maintained for $5 \mathrm{~d}$. On the fifth day, the media was replaced with a $0.01 \% 10,000$ MW dextran conjugated to Texas Red (Molecular Probes) solution in fresh media. In some cases, this media was removed after 30 min and replaced with fresh media for 1 additional hour. Some cultures were kept at $4^{\circ} \mathrm{C}$ as a control for active endocytosis. The cultures were rinsed in PBS, fixed in 4\% paraformaldehyde in PBS, and processed for $\beta$-tubulin III immunohistochemistry as described above. Randomly selected growth cones were imaged, and any growth cone that contained at least five dextran beads was counted as being positively labeled [growth cones on laminin $(n=15)$, growth cones on aggrecan-laminin incubated at $4^{\circ} \mathrm{C}(n=14)$, growth cones on aggrecan-laminin incubated at $37^{\circ} \mathrm{C}$ $(n=27)]$.

Dextran labeling of dystrophic endings in vivo. Lesions were made $1 \mathrm{~mm}$ deep into the thoracic region of the dorsal columns of anesthetized adult Sprague Dawley rats using scissors. Either $1 \mathrm{~d}$ or 1 week after the lesion, the area was surgically reopened very carefully so that the spinal cord was not reinjured. A pulled glass pipette containing $3 \mu \mathrm{l}$ of 10\% 10,000 MW dextran conjugated to Texas Red in saline was carefully lowered into the upper lesion cavity. The dextran solution was dripped very gently onto the cord with a glass pipette. The next day, the rats were anesthetized with isoflurane and perfused transcardially with $0.1 \mathrm{~m}$ PBS followed by $4 \%$ paraformaldehyde and $0.5 \%$ glutaraldehyde in $0.1 \mathrm{M}$ PBS. The spinal cord segment containing the lesion was dissected out, postfixed in $4 \%$ paraformaldehyde overnight at $4^{\circ} \mathrm{C}$, and cut in $40 \mu \mathrm{m}$ sections on a vibratome. The sections were mounted in Citifluor, coverslipped, and sealed with nail polish. 

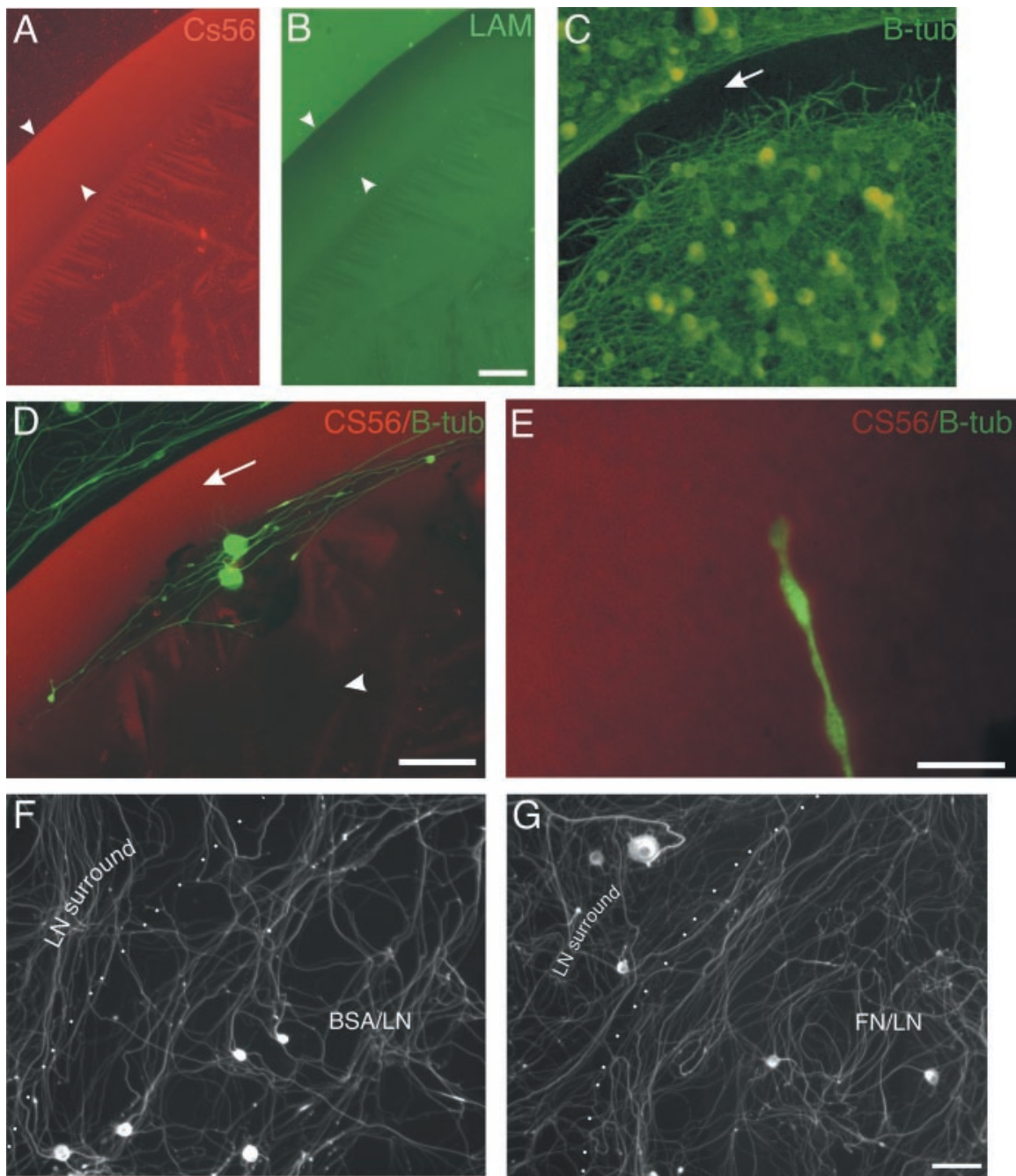

Figure 1. Aggrecan-laminin spot gradient causes the formation of dystrophic endballs. A, A low magnification image of the aggrecan-laminin spot showing the distribution of the aggrecan as visualized via CS56 staining (red). The proteoglycan concentration is lower in the center of the spot and higher in the rim (area between both arrowheads). $B$, The laminin, however, has the opposite pattern (green), and its deposition is greatest in the center of the spot and least at the periphery. $C, \beta$-tubulin III immunohistochemistry of a high-density dissociated adult DRG culture on the aggrecan-laminin spot. There are virtually no neurites in the outermost part of the inhibitory rim, but many that struggle into the inner part of the rim (arrow). D, A lowerdensity culture of DRG neurons (in green via $\beta$-tubulin staining) on the aggrecan-laminin spot (aggrecan in red via CS56 staining). There are fewer neurons growing in the center of the spot (arrowhead), and although there are some in the inner portion of the rim, no neurites are in the outermost rim (arrow). $E$, The appearance of a club ending on a neurite growing in the inner portion of the rim of the aggrecan-laminin spot ( $\beta$-tubulin in green; $C S 56$ in red). $F, G, \beta$-tubulin III staining of DRGs on control spots of either BSA and laminin $(F)$ or fibronectin and laminin $(G)$. Neurites are able to grow well and cross the rims of both spots, demarcated by the dashed lines, into the laminin surround. Scale bars: $A, B, F, G, 50 \mu \mathrm{m} ; D, 30 \mu \mathrm{m} ; E, 20 \mu \mathrm{m}$.

\section{Results}

Characterization of the aggrecan-laminin spot gradient

We have tested the hypothesis that PGs can induce the so-called dystrophic state in axons if the inhibitory matrix is presented in a spatial organization that more closely resembles that which develops after lesions in vivo. To do this, spots of a solution of the PG aggrecan and the growth-promoting molecule laminin were placed on nitrocellulose coverslips and air dried.

A consistent artifact of drying produced a crude gradient in which the rim of the spot contained an increasingly higher concentration of aggrecan than in the center, as shown with CS56 immunohistochemistry (Fig. $1 A$, arrowheads). Interestingly, the very outermost part of the rim contained a lower concentration of laminin than any more central region (Fig. $1 B$, arrowheads).
The optimal ECM concentrations (0.7 $\mathrm{mg} / \mathrm{ml}$ aggrecan and $5 \mu \mathrm{g} / \mathrm{ml}$ laminin) resulted in good cell attachment, albeit with limited outgrowth, which was ideal for generating this particular model of the glial scar. Thus, the high aggrecan-low laminin outer rim appeared to be a particularly harsh terrain for regenerating neurites. None entered inward into the spot from the laminin surround by crossing its sharp outer interface. Fibers growing centripetally from within the center of the spot were able to enter the inner portion of the rim but could grow no farther. Once within the gradient, axons appeared trapped. The curious and reproducible pattern of outgrowth that occurred on this substrate was clearly demonstrated in high-density cultures (Fig. 1C). Lowerdensity cultures (the standard for the remainder of the experiments) in which axon-axon interactions were diminished also resulted in trapped endings, although at a reduced frequency (Fig. 1D). Importantly, club-like, dystrophic endballs (Fig. $1 E$ ) formed at the ends of neurites within the gradient. Moreover, DRGs growing on spots of laminin mixed with either BSA (Fig. $1 F$ ) or fibronectin (Fig. $1 G$ ), where the laminin pattern was identical to that of the aggrecan-laminin spots (data not shown), did not display dystrophic endings, suggesting that dystrophy may be a specific result of the presence and distribution of PGs rather than merely an effect of mixing two proteins in an air-dried spot.

We did not attempt to impose any changes in the patterning of ECM distribution, which forms as a purely fortuitous consequence of drying, other than by altering the various ratios of aggrecan versus laminin. Interestingly, we were able to determine that gradually increasing the initial concentrations of laminin $(5,10$, or 25 $\mu \mathrm{g} / \mathrm{ml}$ ) relative to a fixed concentration of aggrecan $(0.7 \mathrm{mg} / \mathrm{ml})$ eventually allowed many neurites to escape from the confines of the spot (at the $25 \mu \mathrm{g} / \mathrm{ml}$ concentration; $p<0.01$ ) (Fig. $2 B, F-H$ ). Large variations in aggrecan concentration (on the order of many hundreds of micrograms) were also effective in altering axonal patterning in our model; however, because of marked variations in inhibitory potency between different lots of aggrecan, which became especially noticeable at low concentrations, it was necessary to use a relatively high amount to stabilize the aggrecan effect. The use of $0.7 \mathrm{mg} / \mathrm{ml}$ gave us the most consistent results from batch to batch. To determine whether augmenting laminin affected the overall characteristics of the ECM gradient (Fig. 2A), spots containing $0.7 \mathrm{mg} / \mathrm{ml}$ aggrecan and 5, 10, or $25 \mu \mathrm{g} / \mathrm{ml}$ laminin were stained with the CS56 antibody (recognizing 4- and 6-sulfated PGs) and an anti-laminin-1 antibody. Average pixel intensity of both stains was measured every $10 \mu \mathrm{m}$ from the outermost edge 
of the spot inward for a total of $70 \mu \mathrm{m}$ (the width of the rim) (Fig. 2C-E). Curiously, although more laminin was deposited overall as the amount of laminin used increased, the slopes of both the laminin and PG gradients were similar, regardless of the starting laminin concentrations. Thus, from the inner shelf of the rim outward toward the edge of the spot at all three laminin concentrations, there was a gradual and consistent $\sim 35 \%$ increase in pixel intensity of CS56 staining and a 50\% decrease in pixel intensity of laminin staining; however, there was an obvious shift in the ratio of aggrecan to laminin as laminin was increased. This ratio decreased from $1.4(5 \mu \mathrm{g} / \mathrm{ml}$ of laminin $)$ to $1.0(10 \mu \mathrm{g} / \mathrm{ml}$ laminin) to $0.8(25 \mu \mathrm{g} / \mathrm{ml}$ laminin $)$ at the innermost portion of the rim, and from 4 to 3 to 2.3 at the outermost edge of the spot, where CS56 staining is brightest and laminin staining is weakest. Thus, growth cones are exposed to more laminin per aggrecan molecule as initial laminin concentrations are increased.

\section{Time-lapse microscopy of dystrophic endings demonstrate that they are dynamic}

To observe the behavior of the "dystrophic endings" formed in our assay, we used time-lapse microscopy. Dystrophic growth cones growing on aggrecan-laminin gradients were imaged and compared with control growth cones growing on laminin alone. We found that, in vitro, the so-called sterile endings were actually quite dynamic. Growth cones growing on laminin had a relatively small body, with multiple highly active filopodia (Fig. $3 A$ ). They grew at a steady rate of $\sim 25 \mu \mathrm{m} / \mathrm{hr}$ (Fig. 3B). Growth cones growing on aggrecan-laminin gradients were remarkably different. This is evident when a scanning electron microscope image of the dystrophic growth cone (Fig. $3 E$ ) is compared with that of a control growth cone on laminin (Fig. 3D). Furthermore, dystrophic endballs were bulb shaped or club-like, or both (Fig. $3 I-K)$. They often, but not always, completely lacked filopodia, and those that did form were abnormal (Fig. 3E, arrow; see Fig. $5 B$, arrowhead). Many dystrophic endings contained multiple swollen vesicles (Fig. $3 I-K$ ). These club-like, vesicle-containing growth cones were very similar in morphology to those that Ramón y Cajal (1928) described in the lesioned spinal cord (Fig. $3 C)$. Although the morphology of these endings was stereotypically dystrophic, they were far from "sterile." Rather, they were quite dynamic, constantly moving in place as they protruded and withdrew undulating, sheet-like veils (see supplemental Fig. 2 and compare with time-lapse movie of growth cone on laminin in supplemental Fig. 1, available at www.jneurosci.org/cgi/content/ full/24/29/6531/DC1). This cycle repeated itself continuously for many hours, without net forward progress of the growth cone (Fig. $3 B)(p<0.00001)$. Dystrophic growth cones often managed to advance short distances, but inevitably, the struggling growth cone would round up into a more compact ball and retract, only to start moving again. This abundant local motility, as indicated by their to and fro movements and veil-forming behavior, was
B

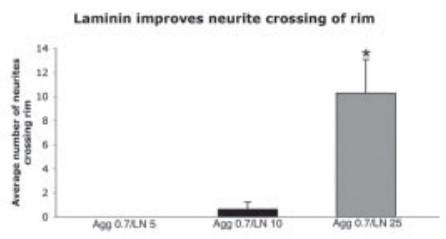

D $0.7 \mathrm{mg} / \mathrm{ml}$ aggrecan, $10 \mathrm{ug} / \mathrm{ml} \mathrm{LN}$
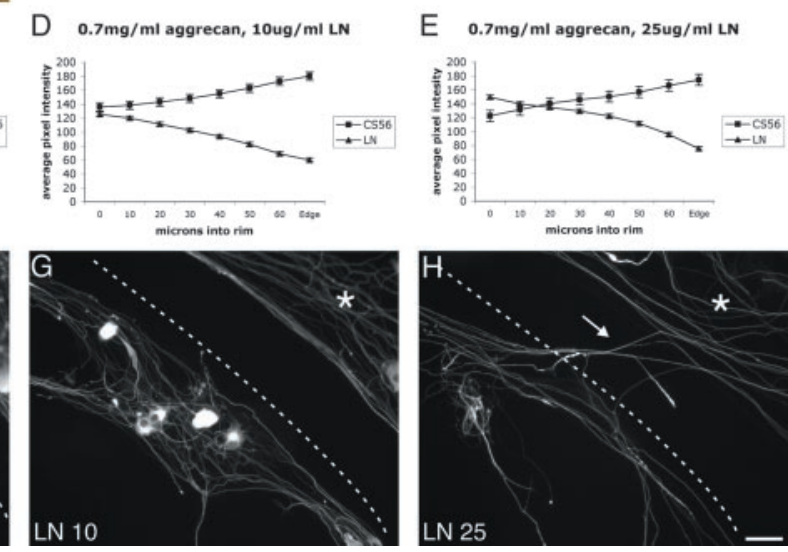

Figure 2. Increasing laminin concentration modifies the aggrecan gradient and allows neurite crossing of the inhibitory rim. $A$, A mage of an aggrecan-laminin spot double stained for CS56 (red) and laminin (green), again demonstrating thei portion, with some crossing of the spot when $10 \mu \mathrm{g} / \mathrm{ml}$ laminin was used, and many fibers crossing when $25 \mu \mathrm{g} / \mathrm{ml}$ was used.

carefully quantified and charted for three different dystrophic growth cones over a $2 \mathrm{hr}$ period (Fig. $3 \mathrm{~F}-\mathrm{H}$ ). Although all three were able to grow past their initial locations, extending (Fig. $3 F-H$, asterisks) and withdrawing (Fig. $3 F-H$, squares) membrane veils throughout, there was an overall net retraction for all three. Examples of their many different and rapidly changing shapes are also shown (Fig. $3 I-K)$.

Time-lapse observations of vesicle dynamics in the dystrophic growth cone

To better understand membrane and vesicle dynamics in the dystrophic growth cone, we attempted to track the paths of some of the most swollen (and, thus, most easy to follow) vesicles in our time-lapse movies. We wished to determine where vacuoles formed, whether they remained in place or changed location, and how long they persisted. Although it turned out to be quite difficult to follow single vesicles even within very short time frames ( 1 min intervals), because of the extremely dynamic nature of the dystrophic endball, we were able to find a few vesicles that could be followed along their entire course. One particular growth cone was especially useful because it had developed notably large membrane bubbles. The vesicle highlighted in Figure 4 (highlighted in red) formed at the leading edge of the growth cone and was gradually transported retrogradely into the core of the endbulb, where it remained for $\sim 10$ min before it disappeared from view. On average, the large membrane blebs persisted between $\sim 10$ and $30 \mathrm{~min}$. The general tendency of vesicles to form at the leading tip of the dystrophic growth cone and move rapidly rearward can be 

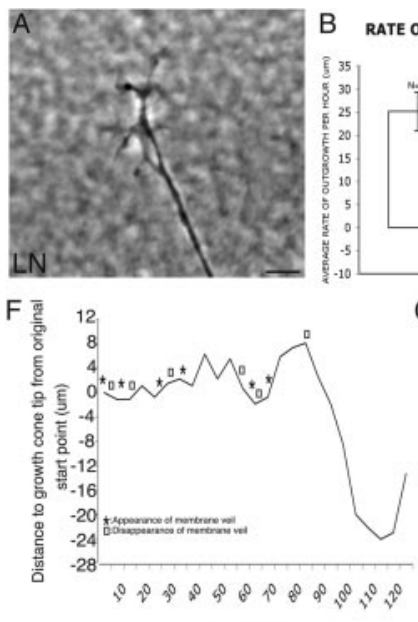

Time (minutes)

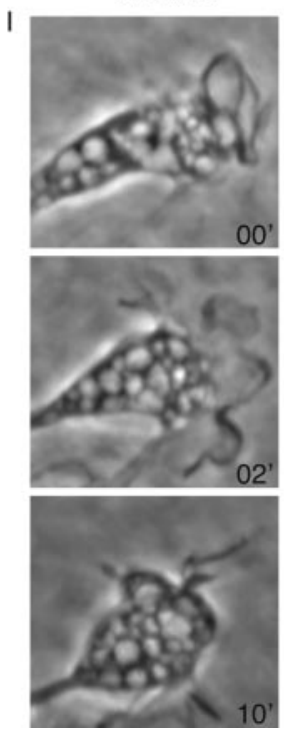

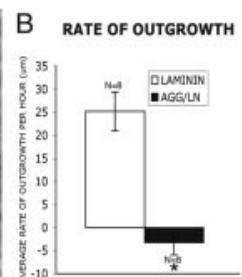

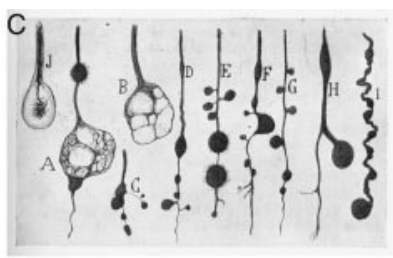

$\mathrm{G}_{4}$

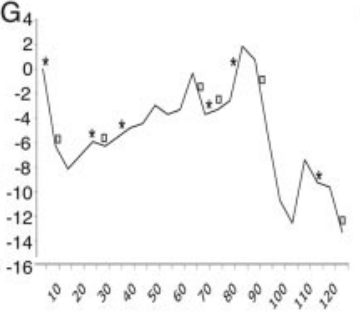

Time (minutes)
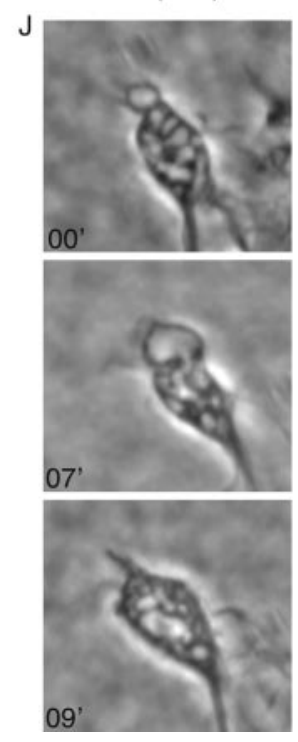

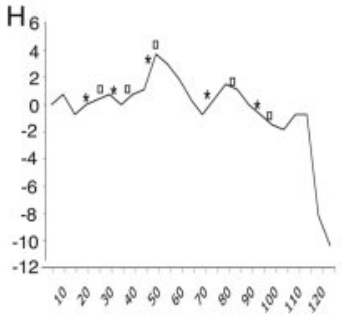

Time (minutes)

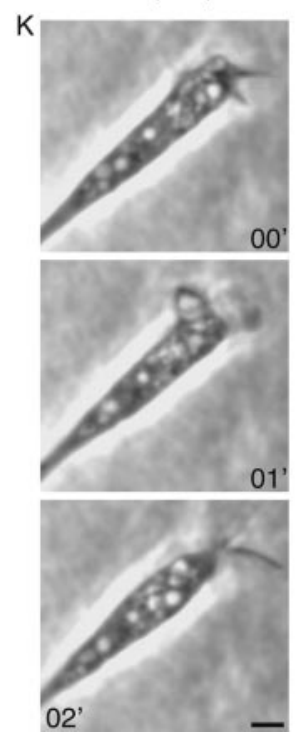

Figure 3. Dystrophic growth cones are dynamic. $A$, Selected frame from a time-lapse microscopy movie of an adult DRG growth cone on laminin only. The growth cone looked stereotypical of those that grow rapidly in that they contain multiple filopodia. $B_{\text {, }}$ Quantification of the rate of neurite outgrowth on either laminin only or aggrecan-laminin. Net advancement of growth cones on laminin $(n=8)$ and aggrecan-laminin $(n=8)$ was quantified and compared. Although growth cones on laminin displayed a net outgrowth forward, there was a net retraction of growth cones on aggrecan-laminin, although they were highly active ( $p<$ 0.0001). C, Drawings by Ramón y Cajal (1928) (reprinted with permission) of dystrophic endings near a lesion site in vivo look like those in the rim in vitro. D, E, Scanning electron microscopy of a control growth cone on laminin $(D)$ and a dystrophic one on aggrecan-laminin (E). The growth cone on laminin was flattened and sent out long thin filopodia $(D$, arrow). The dystrophic endings contained many membrane ruffles ( $E$, arrow) and looked entirely different from growth cones on laminin. $F-H$, Quantification of the dynamism displayed by growth cones (I-K, respectively) on aggrecan-laminin. Time-lapse movies were made of three different endings on the aggrecan-laminin spots. The distance from the distal-most tip of the growth cone from the initial point was measured every $5 \mathrm{~min}$ for a total elapsed period of $2 \mathrm{hr}$. Moreover, the appearance (asterisk) or disappearance (open square) of a membrane veil was also noted. Note that although all three growth cones were able to extend past their initial points, there was a net retraction. $I-K$, Examples of the multiple shapes displayed by the three different growth cones. Even within short time frames, the same growth cone looked completely different, demonstrating that they are extremely dynamic. Scale bars: $A, 10$ $\mu \mathrm{m} ; D, E, 1 \mu \mathrm{m} ; I-K, 5 \mu \mathrm{m}$.

appreciated more readily in high-speed, time-lapse movies (see supplemental Fig. 3, available at www.jneurosci.org/cgi/content/full/24/29/ $6531 / \mathrm{DC} 1)$. Although the exact fate of the vesicles is unknown (i.e., whether they are recycled locally or retrogradely transported into the axon), it did appear that the vesicles were resorbed mainly at the very rear of the growth cone or near the tip of the axon shaft.

\section{In vitro labeling of dystrophic endings with dextran}

Because the endings of dystrophic growth cones are dynamic, albeit relatively stationary, and contain large vacuoles that form at the tip, it is likely that there may be incessant membrane turnover. To determine whether the constantly erupting membrane protrusions on dystrophic growth cones are, in fact, being taken back up into the growth cone, we performed a dextran uptake experiment. Five days after DRGs were plated on the aggrecan-laminin spot gradient, the media was replaced with media containing 10,000 MW dextran-Texas Red for 30 min. The media was then replaced with fresh media for another hour. If the growth cones are actively resorbing membrane, they should take up the dextran.

Very few growth cones on laminin alone ( 3 of 15 , or $20 \%$ ) contained dextran particles (Fig. 5A), and this was at the low end of the positive labeling threshold (at least five particles per growth cone). On the other hand, 23 of 27 dystrophic growth cones $(85.2 \%)$ colocalized with dextran, suggesting uptake (Fig. 5C, arrow). The dextran appeared to be localized mainly in or near the tips of the growth cones, suggesting that there may be local recycling of membrane, at least in the short term. Furthermore, no labeling was seen in 14 of 14 (100\%) dystrophic endings when the cultures were incubated with dextran at $4^{\circ} \mathrm{C}$ for $30 \mathrm{~min}$ (Fig. $5 \mathrm{~B}$ ), suggesting that the dextran seen in dystrophic endings when incubated at $37^{\circ} \mathrm{C}$ (Fig. 5C) was actively taken up and not merely adhering passively to the membrane. Numbers of dextran particles within the individual growth cones are shown (Fig. 5D), giving further quantitative evidence that there is enhanced endocytosis in dystrophic growth cones.

To determine whether the disparate labeling between control and dystrophic growth cones was caused by relatively increased uptake in dystrophic endings versus faster retrograde transport mechanisms in growth cones growing on laminin alone, dextran was placed into the media for $30 \mathrm{~min}$, but cultures were then immediately washed and fixed. If the lack of dextran particles in control growth cones is caused by a fast transport mechanism [fast retrograde transport occurs at a rate of 200 $\mathrm{mm} / \mathrm{d}$ (Jacobson, 1991)], dextran should be found within the growth cone when there is not sufficient time for transport to have cleared it from the growth cone completely. This was not seen, however, and clear differences in labeling remained between control and dystrophic growth cones (data not shown). This suggests that dystrophic endballs in vitro are not only active, but that they also display enhanced endocytosis.

\section{In vivo labeling of dystrophic endings with dextran}

Although dystrophic endings within our in vitro model of the glial scar are active, it does not necessarily follow that those in vivo are 
also active. It would be extremely interesting if they were. Therefore, in an experiment similar to that which we did in vitro, we attempted to label potentially active dystrophic endballs with dextran-Texas Red in vivo. It is known that recently injured axons label with dextran when the dye is presented immediately after injury (Lu et al., 2001). Thus, to ensure that dextran found inside dystrophic endings was caused by an active uptake process rather than an injury response, stab lesions were made in the dorsal columns, and either $1 \mathrm{~d}$ or 1 week later, $10 \%$ dextran in physiological saline was carefully dripped (to avoid reinjuring previously injured axons or injuring uninjured ones) into the upper portion of the lesion cavity.

Similar to what was found in vitro, axonal endings in the dorsal columns were brightly labeled with dextran both $1 \mathrm{~d}$ (Figs. 6A,B, arrows) and 1 week (Figs. $6 C, D$, arrows) after the lesion. Some of the endings appeared to be uniformly labeled, whereas in others, especially those within the 1-week-old lesions, labeling appeared to be more punctate (Figs. 6C,D). Although there were some fibers that were labeled rostral to the lesion (these are likely from the caudally projecting axon population in the dorsal columns; our unpublished observation), most of the labeled axons were located caudal to, and sometimes up to $1 \mathrm{~mm}$ away from, the injury site. White matter tracts distant from the initial lesion were unlabeled. These results suggest that dystrophic endings in vivo are also quite active, at least for 1 week after injury. To determine more accurately the long-term behavior of dystrophic endings, we would have to look at post-lesion times $>1$ week. Because of the extensive accumulating scar, however, it is technically not feasible to drip dextran into the lesion area without first tearing into the tissue, resulting in a new injury that would inevitably lead to false positive data.

\section{Further characterizations of the dystrophic ending}

We also examined several other characteristics of dystrophic growth cones formed in our in vitro assay as well as in vivo after a spinal cord injury. We found that these endings contained an abnormal cytoskeletal arrangement (see supplemental Figs. 4, 5, available at www.jneurosci.org/cgi/content/full/24/29/6531/ DC1). In control growth cones, there was a general, although not complete, separation of tubulin and actin, with tubulin located mainly in the more central palm of the growth cone and F-actin located throughout the growth cone, including the dynamic filopodia in the periphery. In bulbous endings on aggrecan-laminin gradients, however, both $\mathrm{F}$-actin and $\beta$-tubulin III were highly expressed throughout the growth cone, with large amounts of both proteins present in the tip and in their rare, misshapen filopodia (Fig. 5B, arrowhead). Also, we demonstrated that dystrophic endings in vitro and for a period in vivo after spinal cord injury ectopically express integrins all over their surfaces (see supplemental Figs. 6, 7, available at www.jneurosci.org/cgi/ content/full/24/29/6531/DC1). Furthermore, we compared
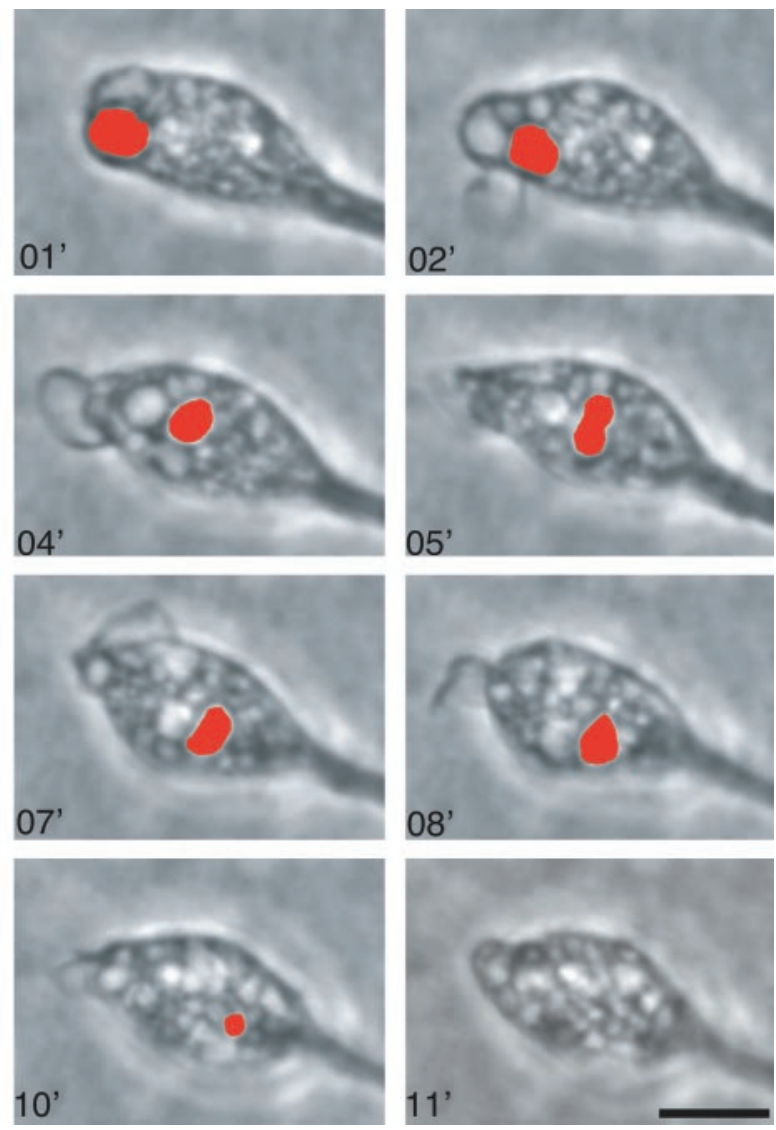

$11^{\prime}$

A single vesicle (highlighted in red) was tracked in individual image files taken from a time-lapse movie of a growth cone on aggrecan-laminin from its initial appearance at the front of the growth cone over a period of $12 \mathrm{~min}$, when the vesicle could no longer be readily identified. During that time, the vesicle gradually moved toward the rear of the growth cone. Scale bar, $5 \mu \mathrm{m}$.

proteoglycan-induced dystrophy with myelin membraneinduced collapse of growth cones, a hypothesized mechanism for the failure of regeneration in the adult CNS (see supplemental Fig. 8, available at www.jneurosci.org/cgi/content/full/24/29/ 6531/DC1).

\section{Discussion}

We developed an in vitro assay that mimics in two-dimensions the PG gradient phenotype that exists in the glial scar. Fully adult DRGs form dystrophic endings in vitro that are morphologically and behaviorally similar to those that form in the vicinity of the glial scar in vivo. Our studies of these unusual endings demonstrate, for the first time, that they can be quite dynamic both in vitro and in vivo, suggesting that they may be more capable of being reawakened into a regenerative state than was believed previously.

The dynamic behavior of dystrophic endballs is closely associated with the formation of large membrane vesicles. Although these huge vacuoles make the dystrophic growth cone appear highly abnormal, vesicles in retracting growth cones have also been documented (Cheng and Reese, 1987; Dailey and Bridgman, 1993; Fournier et al., 2000), suggesting that dystrophy and collapse may be similar, with dystrophy being on the more extreme end of a continuum of growth cone response to inhibitory cues. Such vesicles, although considerably smaller than the largest ones seen in dystrophic growth cones [500 nm (Dailey and Bridgman, 1993) compared with up to $2-3 \mu \mathrm{m}$ in dystrophic growth cones] were hypothesized to be part of a turnover mechanism 

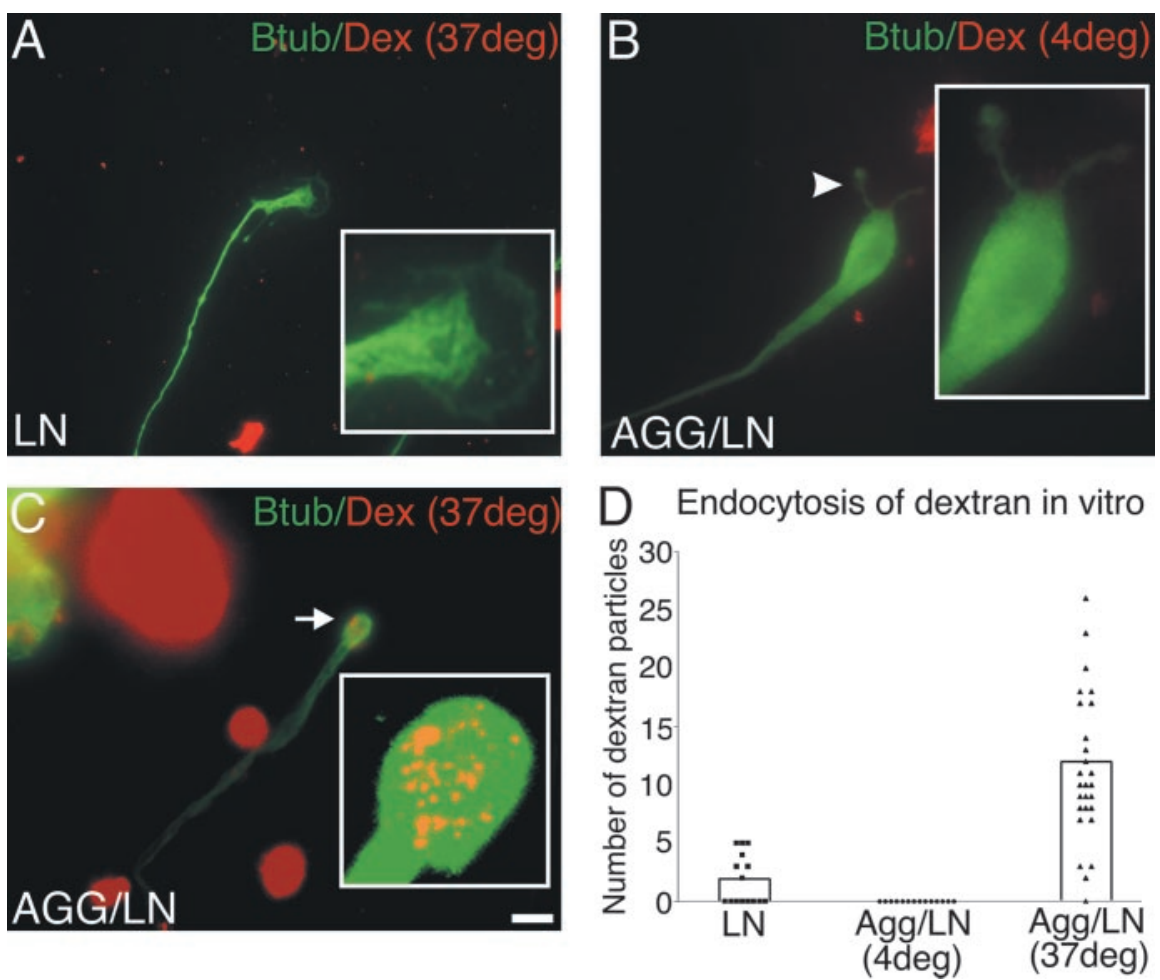

Figure 5. Uptake of dextran by dystrophic endings demonstrates activity in vitro. After $5 \mathrm{~d}$ in culture, cultures were exposed to dextran-Texas Red for 30 min followed by $1 \mathrm{hr}$ wash. $A, A \beta$-tubulin III-positive (green) control growth cone growing on laminin does not endocytose much dextran (red). $B$, A dystrophic growth cone grown on aggrecan-laminin for $5 \mathrm{~d}$ that was then incubated with dextran-Texas Red for $30 \mathrm{~min}$ at $4^{\circ} \mathrm{C}$. No dextran was taken up under these conditions. A $\beta$-tubulin-rich filopodia is highlighted by the arrowhead (see Discussion). C, An example of a dystrophic growth cone that had ingested dextran (arrow). Inset is a high-magnification confocal image of the same growth cone. Because no dextran was taken up by dystrophic endings at $4^{\circ} \mathrm{C}$, this suggests that the dextran seen in $C$ is from an active uptake process and not caused by dextran passively attaching to the surface of the growth cone. $D$, The numbers of dextran particles in individual growth cones show obvious differences in uptake between control and dystrophic endings. The averages are depicted by the bars $\left({ }^{*} p<0.01\right)$. Scale bar, $10 \mu \mathrm{m}$.

that allows for endocytosis of membrane that is added but not subsequently used because the growth cone is not moving forward. Membrane recycling may provide a mechanism that allows the dystrophic growth cone to continually deliver novel membrane to its tip while attempting to advance; however, the finding that dystrophic endballs have an exaggerated rate of endocytosis and that this process apparently occurs at the peripheral domain of the growth cone may violate the tenets of normal membrane dynamics, resulting in a state that is exceptionally nonconducive for elongation.

In properly advancing growth cones, membranes associated with cell adhesion molecules (CAMs), such as L1, are usually endocytosed in the central (but not the peripheral) domain and reinserted at the tip of the peripheral domain during outgrowth (Kamiguchi et al., 1998; Kamiguchi and Lemmon, 2000). This polarized recycling of membrane has also been demonstrated with integrin receptors (Lawson and Maxfield, 1995), suggesting that such tractor-like membrane motility may be a basic mechanism necessary for growth cone advancement. Although it has yet to be determined precisely where membrane is reincorporated within the dystrophic growth cone, it may be that membrane is reinserted at the rear, because the vesicles in dystrophic endings seem to vanish there, rather than at the leading edge, contradicting normal membrane trafficking patterns. Following small labeled patches of membrane with a fluorescent lipid analog (i.e., DiI) (Popov et al., 1993) or tracking lipid-attached beads (Dai and Sheetz, 1995) over time would be needed to determine the ultimate fate of endocytosed membrane.

One theory for filopodial protrusion and growth cone advance, known as the "clutch" hypothesis, surmises that growth cone extension occurs when molecules that interact with the substrate, such as integrins and CAMs, are able to form a firm linkage to the cytoskeleton (the clutch). In particular, linkage with the F-actin network is thought to be especially critical because of its ability to create tension required for movement (Mitchison and Kirschner, 1988; Jay, 2000; Suter and Forscher, 2000; Dent and Gertler, 2003). The three variables in the hypothesis, actin assembly, engagement-disengagement of the clutch, and retrograde flow of F-actin, are "summed" to produce a net effect on the growth cone ranging from rapid extension to rapid retraction, with stationary states in between. It has been demonstrated that growth cone advancement and retrograde flow of F-actin are inversely correlated (Lin and Forscher, 1995), lending support to the hypothesis. It is likely that several of the behavioral and molecular oddities that we have observed in the dystrophic growth cone may be acting to stall forward movement by chronically disengaging the clutch. For instance, the abnormal, widely dispersed integrin arrangement that we observed in dystrophic endballs (see supplementary data, available at www.jneurosci.org/cgi/content/ full/24/29/6531/DC1) could interfere with proper clutch engagement and thus diminish tension-producing interactions with cytoskeletal-linking proteins, especially if these critical substrate receptors have become unstable because of the highly fluid nature of the outer membrane of the dystrophic growth cones.

The large amount of retrograde membrane movement in the dystrophic growth cone may also be intimately associated with the unusual arrangement of its cytoskeletal network. The attenuation of retrograde F-actin flow has been linked through an unknown mechanism to the invasion of microtubules farther into the growth cone to the area that is depleted of actin (Suter et al., 1998). Likewise, when growth cones are treated with cytochalasin, microtubules enter the F-actin-depleted region (Forscher and Smith, 1988). Why microtubules extend abnormally to the most distal ends of the dystrophic growth cone (see supplementary data, available at www.jneurosci.org/cgi/content/full/24/29/ $6531 / \mathrm{DC} 1$ ) and even into their uncommon filopodia (Fig. $5 B$, arrowhead) is unknown, but their presence distally may be necessary to provide sufficient internal structural support that is required of an unusually motile but poorly adherent axon tip. Whether there is a disruption in the retrograde movement of F-actin that accompanies the increase in membrane turnover in dystrophic endings is unknown, but such cytoskeletal abnormalities would only serve to further render the clutch inoperable. It would be interesting and potentially therapeutic to determine whether experimental manipulation of the three components of 

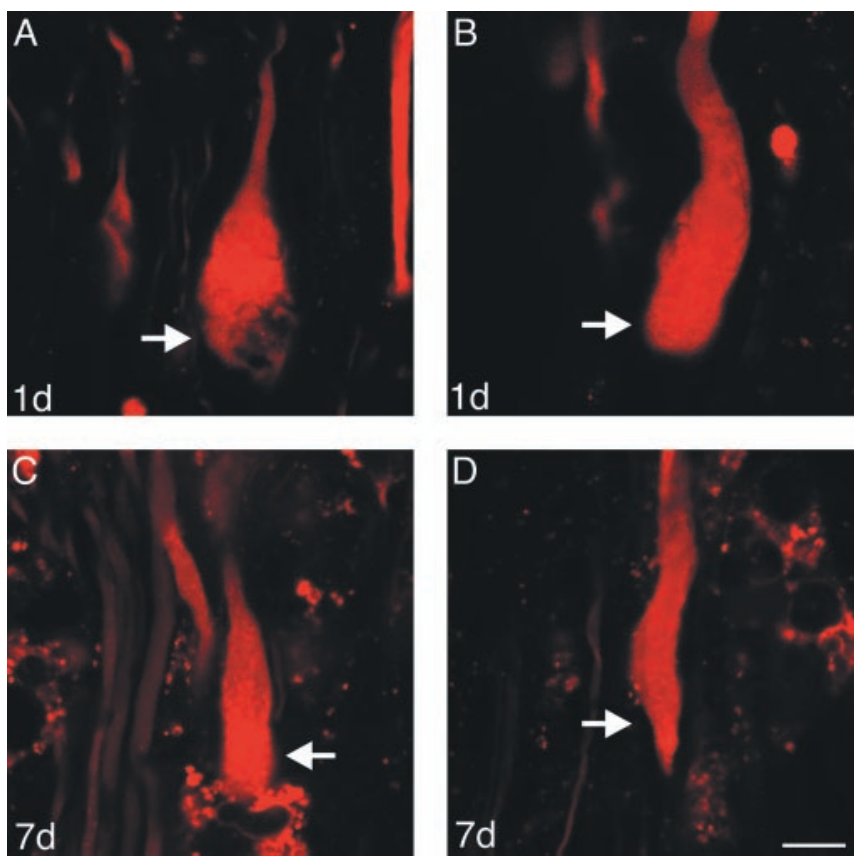

Figure 6. Dextran uptake by dystrophic endings demonstrates activity in vivo well after injury. $A, B$, Confocal images of dystrophic endings of axons (arrows) in spinal cord that were able to take up dextran-Texas Red $1 \mathrm{~d}$ after a lesion. C, D, Confocal images of dystrophic endings (arrows) of spinal cord axons that took up dextran-Texas Red $7 \mathrm{~d}$ after lesion. Some of these active endings contain large vacuoles $(A, C)$. Scale bar, $10 \mu \mathrm{m}$.

the clutch would have any effect on improving the behavior of dystrophic growth cones in our assay.

One important avenue of research yet to be addressed is the identification of the particular signaling cascades that are active in the trauma-induced dystrophic state. Studies have implicated focal adhesion kinase (FAK) signaling pathways in dystrophic neurites in an in vitro model of Alzheimer's disease (Grace and Busciglio, 2003); however, neuritic dystrophy in that model consisted of kinked, tangled neurites rather than neurites that had a grossly swollen endball. Therefore, it is not known whether FAK is playing a role in the more obvious form of dystrophy. Recent studies have also implicated phosphatidylinositol 3-kinase and mitogen-activated protein kinase kinase (Atwal et al., 2003), rhofamily GTPases (Jin and Strittmatter, 1997; Kuhn et al., 1999; Lehmann et al., 1999; Västrik et al., 1999; Dergham et al., 2002; Niederost et al., 2002; Fournier et al., 2003), and cyclic nucleotides (Song et al., 1998; Cai et al., 2001) in semaphorin- or myelin protein-induced collapse-inhibition of growth cones. Interestingly, inhibiting rho-kinases and increasing cyclic nucleotide levels have only a minimal effect on increasing neurite outgrowth across the rim in our model (our unpublished observations). Learning which signaling mechanisms may be going awry in dystrophic axons may help delineate other potential therapeutic paths.

In conclusion, we have developed a novel in vitro model of the glial scar that causes classically dystrophic endballs to form on adult DRG axons. Although their struggle for advancement is often in vain, they are constantly turning over membrane and apparently trying to advance, suggesting that regeneration across the scar may be successful if we can learn how to properly harness their growth potential while also modifying the negative aspects of scarring. We now have a better understanding of what a dystrophic growth cone is and why it forms. Importantly, our data suggest that it may not simply be the excess of inhibitory matrices at CNS lesions that leads to regeneration failure and dystrophy but, rather, that their distribution in a gradient transforms the growth cone into a state that is apparently incapable of freeing itself from the lesion environment. Finally, it should be noted and stressed that although we have learned that an appropriately crafted aggrecan gradient in combination with laminin is sufficient to create growth cone dystrophy in an in vitro setting, we do not yet know what roles other potential inhibitors, such as semaphorins, ephrins, slits, and other types of proteoglycans, which are all present in the scar and could also be patterned in a gradient (Zhang et al., 1997; Miranda et al., 1999; De Winter et al., 2002; Bundesen et al., 2003; Hagino et al., 2003; Tang et al., 2003), may play in triggering the dystrophic state in vivo. We now look toward developing and screening possible therapies that allow would-be dystrophic axons to regenerate robustly past the outermost rim in our glial scar model and then determining whether the optimal strategy translates in vivo.

\section{References}

Atwal JK, Singh KK, Tessier-Lavigne M, Miller FD, Kaplan DR (2003) Semaphorin 3F antagonizes neurotrophin-induced phosphatidylinositol 3-kinase and mitogen-activated protein kinase kinase signaling: a mechanism for growth cone collapse. J Neurosci 23:7602-7609.

Bradbury EJ, Moon LD, Popat RJ, King VR, Bennett GS, Patel PN, Fawcett JW, McMahon SB (2002) Chondroitinase ABC promotes functional recovery after spinal cord injury. Nature 416:636-640.

Bundesen LQ, Scheel TA, Bregman BS, Kromer LF (2003) Ephrin-B2 and EphB2 regulation of astrocyte-meningeal fibroblast interactions in response to spinal cord lesions in adult rats. J Neurosci 23:7789-7800.

Cai D, Qiu J, Cao Z, McAtee M, Bregman BS, Filbin MT (2001) Neuronal cyclic AMP controls the developmental loss in ability of axons to regenerate. J Neurosci 21:4731-4739.

Cheng TPO, Reese TS (1987) Recycling of plasmalemma in chick tectal growth cones. J Neurosci 7:1752-1759.

Dai J, Sheetz MP (1995) Axon membrane flows from the growth cone to the cell body. Cell 83:693-701.

Dailey ME, Bridgman PC (1993) Vacuole dynamics in growth cones: correlated EM and video observations. J Neurosci 13:3375-3393.

Davies SJ, Goucher DR, Doller C, Silver J (1999) Robust regeneration of adult sensory axons in degenerating white matter of the adult rat spinal cord. J Neurosci 19:5810-5822.

Dent EW, Gertler FB (2003) Cytoskeletal dynamics and transport in growth cone motility and axon guidance. Neuron 40:209-227.

Dergham P, Ellezam B, Essagian C, Avedissian H, Lubell WD, McKerracher L (2002) Rho signaling pathway targeted to promote spinal cord repair. J Neurosci 22:6570-6577.

De Winter F, Oudega M, Lankhorst AJ, Hamers FP, Blits B, Ruitenberg MJ, Pasterkamp RJ, Gispen WH, Verhaagen J (2002) Injury-induced class 3 semaphorin expression in the rat spinal cord. Exp Neurol 175:61-75.

Fitch MT, Silver J (1997) Activated macrophages and the blood-brain barrier: inflammation after CNS injury leads to increases in putative inhibitory molecules. Exp Neurol 148:587-603.

Fitch MT, Doller C, Combs CK, Landreth GE, Silver J (1999) Cellular and molecular mechanisms of glial scarring and progressive cavitation: in vivo and in vitro analysis of inflammation-induced secondary injury after CNS trauma. J Neurosci 19:8182-8198.

Forscher P, Smith SJ (1988) Actions of cytochalasins on the organization of actin filaments and microtubules in a neuronal growth cone. J Cell Biol 107:1505-1516.

Fournier AE, Nakamura F, Kawamoto S, Goshima Y, Kalb RG, Strittmatter SM (2000) Semaphorin3A enhances endocytosis at sites of receptor-Factin colocalization during growth cone collapse. J Cell Biol 149:411-421.

Fournier AE, Takizawa BT, Strittmatter SM (2003) Rho kinase inhibition enhances axonal regeneration in the injured CNS. J Neurosci 23:1416-1423.

Grace EA, Busciglio J (2003) Aberrant activation of focal adhesion proteins mediates fibrillar amyloid $\beta$-induced neuronal dystrophy. J Neurosci 23:493-502.

Grimpe B, Silver J (2004) A novel DNA enzyme reduces glycosaminoglycan chains in the glial scar and allows microtransplanted dorsal root ganglia 
axons to regenerate beyond lesions in the spinal cord. J Neurosci 24:1393-1397.

Hagino S, Iseki K, Mori T, Zhang Y, Hikake T, Yokoya S, Takeuchi M, Hasimoto H, Kikuchi S, Wanaka A (2003) Slit and glypican-1 mRNAs are coexpressed in the reactive astrocytes of the injured adult rat brain. Glia 42:130-138.

Houle JD, Jin Y (2001) Chronically injured supraspinal neurons exhibit only modest axonal dieback in response to a cervical hemisection lesion. Exp Neurol 169:208-217.

Jacobson M (1991) Developmental neurobiology. New York: Plenum.

Jay DG (2000) The clutch hypothesis revisited: ascribing the roles of actinassociated proteins in filopodial protrusion in the nerve growth cone. J Neurobiol 44:114-125.

Jin Z, Strittmatter SM (1997) Racl mediates collapsin-1-induced growth cone collapse. J Neurosci 17:6256-6263.

Jones LL, Margolis RU, Tuszynski MH (2003) The chondroitin sulfate proteoglycans neurocan, brevican, phosphocan, and versican are differentially regulated after spinal cord injury. Exp Neurol 182:399-411.

Kamiguchi H, Lemmon V (2000) Recycling of the cell adhesion molecule L1 in axonal growth cones. J Neurosci 20:3676-3686.

Kamiguchi H, Long KE, Pendergast M, Schaefer AW, Rapoport I, Kirchhausen T, Lemmon V (1998) The neural cell adhesion molecule L1 interacts with the AP-2 adaptor and is endocytosed via the clathrinmediated pathway. J Neurosci 18:5311-5321.

Kuhn TB, Brown MD, Wilcox CL, Raper JA, Bamburg JR (1999) Myelin and collapsin-1 induce motor neuron growth cone collapse through different pathways: inhibition of collapse by opposing mutants of Rac1. J Neurosci 19:1965-1975.

Kwon BK, Liu J, Messerer C, Kobayashi NR, McGraw J, Oshipok L, Tetzlaff W (2002) Survival and regeneration of rubrospinal neurons 1 year after spinal cord injury. Proc Natl Acad Sci USA 99:3246-3251.

Lawson MA, Maxfield FR (1995) $\mathrm{Ca}^{2+}$ - and calcineurin-dependent recycling of an integrin to the front of migrating neutrophils. Nature 377:75-79.

Lehmann M, Fournier A, Selles-Navarro I, Dergham P, Sebok A, Leclerc N, Tigyi G, McKerracher L (1999) Inactivation of Rho signaling pathway promotes CNS axon regeneration. J Neurosci 19:7537-7547.

Li Y, Raisman G (1995) Sprouts from cut corticospinal axons persist in the presence of astrocytic scarring in long-term lesions of the adult rat spinal cord. Exp Neurol 134:102-111.

Lin C, Forscher P (1995) Growth cone advance is inversely proportional to retrograde F-actin flow. Neuron 14:763-771.

Lu J, Ashwell KW, Hayek R, Waite P (2001) Fluororuby as a marker for detection of acute axonal injury in rat spinal cord. Brain Res 915:118-123.

Mayes DA, Houle JD (2003) Combined use of matrix degrading enzymes and neurotrophic factors to facilitate axonal regeneration after spinal cord injury. Soc Neurosci Abstr 29:245.11.

McKeon RJ, Schreiber RC, Rudge JS, Silver J (1991) Reduction of neurite outgrowth in a model of glial scarring following CNS injury is correlated with the expression of inhibitory molecules on reactive astrocytes. J Neurosci 11:3398-3411.

Miranda JD, White LA, Marcillo AE, Willson CA, Jagid J, Whittemore SR (1999) Induction of Eph3B after spinal cord injury. Exp Neurol 156:218-222.

Mitchison T, Kirschner M (1988) Cytoskeletal dynamics and nerve growth. Neuron 1:761-772.

Moon LD, Asher RA, Rhodes KE, Fawcett JW (2001) Regeneration of CNS axons back to their target following adult rat brain treatment with chondroitinase ABC. Nat Neurosci 4:465-466.

Morgenstern DA, Asher RA, Fawcett JW (2002) Chondroitin sulphate proteoglycans in the CNS injury response. Prog Brain Res 137:313-322.

Niederost B, Oertle T, Fritsche J, McKinney RA, Bandtlow CE (2002) Nogo-A and myelin-associated glycoprotein mediate neurite growth inhibition by antagonistic regulation of RhoA and Rac1. J Neurosci 22:10368-10376.

Popov S, Brown A, Poo M-M (1993) Forward plasma membrane flow in growing nerve processes. Science 259:244-246.

Ramón y Cajal S (1928) Degeneration and regeneration of the nervous system. London: Oxford UP.

Silver J, Miller JH (2004) Regeneration beyond the glial scar. Nat Rev Neurosci 5:146-156.

Snow DM, Lemmon V, Carrino DA, Caplan AI, Silver J (1990) Sulfated proteoglycans in astroglial barriers inhibit neurite outgrowth in vitro. Exp Neurol 109:111-130.

Song H, Ming G, He Z, Lehmann M, McKerracher L, Tessier-Lavigne M, Poo M (1998) Conversion of neuronal growth cone responses from repulsion to attraction by cyclic nucleotides. Science 281:1515-1518.

Suter DM, Forscher P (2000) Substrate-cytoskeletal coupling as a mechanism for the regulation of growth cone motility and guidance. J Neurobiol 44:97-113.

Suter DM, Errante LD, Belotserkovsky V, Forscher P (1998) The Ig superfamily cell adhesion molecule, apCAM, mediates growth cone steering by substrate-cytoskeletal coupling. J Cell Biol 141:227-240.

Tang X, Davies JE, Davies SJA (2003) Changes in distribution, cell associations, and protein expression levels of NG2, neurocan, phosphocan, brevican, versican, $\mathrm{V} 2$, and tenascin-C during acute to chronic maturation of spinal cord tissue. J Neurosci Res 71:427-444.

Tester NJ, Plass AH, Howland DR (2003) Chondroitin sulfate glycosamines and the effects of chondroitinase $\mathrm{ABC}$ on behavioral and anatomical recovery following spinal cord injury in the adult cat. Soc Neurosci Abstr 29:744.17.

Västrik I, Eickhold BJ, Walsh FS, Ridley A, Doherty P (1999) Sema3Ainduced growth-cone collapse is mediated by Racl amino acids 17-32. Curr Biol 9:991-998.

Zhang Y, Winterbottom JK, Schachner M, Lieberman AR, Anderson PN (1997) Tenascin-C expression and axonal sprouting following injury to the spinal dorsal columns in the adult rat. J Neurosci Res 49:433-450. 